\title{
Analysis of thyme essential oils using gas-phase broadband rotational spectroscopy
}

\author{
María Mar Quesada-Moreno, ${ }^{\text {ab }}$ Anna Krin, ${ }^{\text {ab }}$ and Melanie Schnell ${ }^{* a b}$ \\ a Deutsches Elektronen-Synchrotron DESY, Notkestraße 85, 22607 Hamburg, Germany. Email: \\ melanie.schnell@desy.de \\ ${ }^{b}$ Christian-Albrechts-Universität zu Kiel, Institut für Physikalische Chemie, Max-Eyth-Straße 1, 24118 Kiel, \\ Germany \\ $\dagger$ Electronic supplementary information (ESI) available. See DOI: 10.1039/c9cp05583e
}

A semi-quantitative analysis of the components of two natural essential oils has been carried out using broadband rotational spectroscopy, which is inherently molecule specific. The samples under study have been two thyme essential oils from Spain with different compositions: a) with thymol as the most abundant species (thyme I) and b) with linalool and 4-carvomenthenol being the most abundant ones (thyme II). Relative intensity measurements of selected rotational transitions were carried out to estimate the abundances of the different species present in these complex mixtures, taking into account the square of the respective dipole moment components. One strength of rotational spectroscopy is its structure sensitivity. Here, we also re-investigate the microwave spectrum of linalool and determined the accurate experimental gas-phase structures of thymol and linalool through the assignment of all ${ }^{13} \mathrm{C}$ isotopologues of their lowest energy conformers. A characteristic splitting pattern of the rotational transitions due to internal rotation of two nonequivalent methyl groups of linalool was observed in the thyme II spectrum. Their internal rotation barriers were experimentally determined to $4.7703(96) \mathrm{kJ} / \mathrm{mol}$ and $9.2581(74) \mathrm{kJ} / \mathrm{mol}$, respectively.

\section{Introduction}

Terpenes are the major constituents of essential oils, which confer them their chemical and biological activities, such as antibacterial, antimicrobial, antioxidant, anti-inflammatory or analgesic ones, resulting in biomedical and pharmacological interest. ${ }^{1-3}$ They are also used in food (as flavors) and fragrance industries and as natural pesticides and insects repellents. ${ }^{4}$ These terpenes are often structurally similar to one another, like thymol, carvacrol, and 4-carvomenthenol in the case of thyme essential oil, although their biochemical impact can be largely different. ${ }^{5,6}$ They can exist as several conformers and isomers. Their unambiguous identification is complicated because of their structural similarity. These oils are usually enantio-enriched due to their natural origin. ${ }^{7}$ A wellestablished technique to analyze the composition of essential oils is gas chromatography, also coupled with mass spectrometry (GC-MS). Using this technique it is possible to separate complex mixtures, to determine trace amounts of contaminants and to quantify the different analytes present in a sample. A drawback of GC-MS techniques is that it can be difficult to resolve the retention time difference of structurally related components, like the structural isomers of terpenes. ${ }^{8}$ Large progress has been made from packed to capillary to multidimensional gas chromatography, and also with the development of chiral analysis, trying to increase the "separation space" of the analysis. ${ }^{8}$ For chiral gas chromatography analysis, the preparation of the chiral stationary phase can be laborious and time-consuming. In addition, mass spectrometry can be ambiguous for this kind of structurally similar species, being in a quite narrow mass window. ${ }^{8}$ Thus, it is of large interest to also develop complementary techniques for complex mixture analysis. FT-IR spectroscopy has also been used to analyze the composition of essential oils, ${ }^{9}$ also in combination with multidimensional gas chromatography. ${ }^{8}$ 
Rotationally resolved high-resolution molecular spectroscopy offers an inherent advantage for mixture analysis because even very similar molecules can be unambiguously identified and differentiated due to its high structure sensitivity. High-resolution rotational spectroscopy in the gas phase (also denoted as microwave or molecular rotational resonance (MRR) spectroscopy) has been used to study the structures of several monoterpenes, for example linalool, ${ }^{10}$ carvone, ${ }^{11,12}$ fenchone, ${ }^{13}$ camphor $^{14,15}$ or pulegone. ${ }^{16}$ Additionally, the chiral analysis of conformational mixtures $^{12}$ and mixtures of different terpenes ${ }^{17}$ has been demonstrated with a combination of broadband rotational spectroscopy and the chirality-sensitive microwave three-wave-mixing (M3WM) technique. Recently, two studies were reported on using this last experimental approach to analyze commercially available peppermint iil $^{7,18}$ and buchu oil, ${ }^{19}$ identifying the terpenes present in the oils and performing a chiral analysis of menthone and its diastereomer isomenthone in the oils. Rotational spectroscopy is inherently mixture compatible. It has an outstanding structure sensitivity because conformers, diastereomers, structural isomers, and isotopologues present unique rotational spectra. ${ }^{7,18}$ Once a molecule has been detected with rotational spectroscopy, it can unambiguously be assigned again in any mixture via their rotational signatures. Moreover, the samples can be analyzed without preparation and separation, and because of the recent developments in microwave spectroscopy, the introduction of the chirp technique, spectra can be recorded in just a few hours. ${ }^{16}$ As a requirement, the molecules need to have a permanent dipole moment and have to be brought into the gas phase.

Via the detection and analysis of isotopologues in natural abundance in the rotational spectra, the experimental atom positions with respect to the center of mass of the molecule can be determined accurately using the isotopic changes of the moments of inertia due to isotopic substitution by solving the Kraitchman equations. ${ }^{14,20-24}$ The conformational landscape and intramolecular dynamics of a molecule can be analyzed, obtaining for instance information on tunneling pathways and barrier heights. ${ }^{23,25-30}$ With this technique in hand we do not only achieve a chemical analysis of a complex molecular sample, but also accurate molecular structures are obtained.

In this work, we present the analysis of the compositions of the two natural thyme essential oils, thyme I and thyme II, from Spain using broadband rotational spectroscopy. In thyme I, thymol is the most abundant terpene according to the supplier, whereas in thyme II, linalool and 4carvomenthenol are dominating. It stands out that the compositions of two natural thyme essential oils coming from the same country and extracted from the flowers of the same plant species, Thymus vulgaris, are different. However, it was observed before that the chemical composition of essential oils shows variations according to the environment, growth region, and cultivation practices. $^{3}$ For instance, six chemotypes have been reported for Thymus vulgaris, that is, geraniol, linalool, $\gamma$-terpineol, carvacrol, thymol, and trans-thujan-4-ol/terpinen-4-ol, which means that the respective chemical compound is present in majority according to the region where it has been cultivated, among other factors. ${ }^{3}$ In the present work, we will further explore the application of microwave spectroscopy as a chemical analysis tool, building on previous work, ${ }^{18,19}$ and we will demonstrate and discuss some of the strengths and weaknesses of broadband rotational spectroscopy for the analysis of natural essential oils.

\section{Methods}

\subsection{Experimental}

Two thyme essential oils ("Thyme Linalool Organic" and "Thyme Thymol Organic") were purchased from Florihana (100\% organic) and used without further purification. Both were extracted from the flowering tops of Thymus Vulgaris plants from Spain using steam distillation. The supplier provided the gas chromatography analyses of three "Thyme Linalool Organic" samples (lot numbers B280917ES, L280818ES and B230419ES) and two "Thyme Thymol Organic" samples (lot numbers B221217ES and L121018ES) on its webpage, ${ }^{31}$ which are the ones 
we used for the comparisons in Tables 2 and 3 in section 3.1. We do not have the specific gas chromatography analysis of the samples we analyzed. $(R)-(-)$-linalool, used for comparison and a detailed internal rotation analysis, was purchased from Sigma-Aldrich (95\% purity) and used without further purification.

All the broadband rotational spectra were recorded with the Hamburg broadband chirpedpulse Fourier-transform microwave (CP-FTMW) spectrometer COMPACT in the frequency ranges 2-8 GHz for the two thyme oils and $2-18 \mathrm{GHz}$ for pure linalool. ${ }^{32}$ All samples were liquids at room temperature. The boiling points of the different identified terpenes forming these essential oils are summarized in Table 1. We used custom-made sample reservoirs as part of our pulsed valves, located close to the valve orifice and heated to $75^{\circ} \mathrm{C}$ for the pure linalool experiment and to $100{ }^{\circ} \mathrm{C}$ in the case of the thyme oils, to generate sufficient vapor pressure. We also recorded the rotational spectra for thyme I oil at $80^{\circ} \mathrm{C}, 90^{\circ} \mathrm{C}$ and $100^{\circ} \mathrm{C}$, and for thyme II oil at $80{ }^{\circ} \mathrm{C}, 100{ }^{\circ} \mathrm{C}$ and 120 ${ }^{\circ} \mathrm{C}$ to ensure that the temperature does not significantly alter the abundances in the gas phase (see section 3.1.). Neon was used as the carrier gas, with backing pressures of 3 bars (oils) and 2.5 bars (linalool), and flowed through this heated reservoir containing thyme I oil, thyme II oil or linalool, depending on the experiment, where the terpenes were picked up. We used a pulsed valve (General valve Series 9) operated at $9 \mathrm{~Hz}(2-8 \mathrm{GHz}$ spectral range) or $4 \mathrm{~Hz}(8-18 \mathrm{GHz}$ region) to introduce the molecules seeded in the carrier gas into the vacuum chamber. The molecular mixture was supersonically expanded into the vacuum chamber, where it was polarized by a chirp spanning the 2-8 GHz frequency range within $4 \mu \mathrm{s}$. For linalool, we recorded the 8-18 GHz range using chirps of $2.5 \mathrm{GHz}$ spectral width. The microwave chirps were created by an arbitrary waveform generator (AWG), amplified with a $300 \mathrm{~W}$ traveling wave tube amplifier $(2-8 \mathrm{GHz})$ or a $50 \mathrm{~W}$ solid-state amplifier $(8-18 \mathrm{GHz})$ and then broadcasted into the vacuum chamber using a horn antenna. The molecular signal was collected in the time domain as a free induction decay (FID). Fast Fourier transformation of the FID gave us the rotational spectrum. The experimental setup is described in detail in ref. 32. The "fast frame" option of the digital oscilloscope was used for these experiments. $^{33}$ Eight back-to-back excitation chirps were performed per gas pulse, and the subsequent eight FID acquisitions were co-added and averaged. In this way, the measurement time and sample consumption were decreased, resulting in an effective repetition rate of $72 \mathrm{~Hz}(2-8 \mathrm{GHz}$ spectral range) or $32 \mathrm{~Hz}(8-18 \mathrm{GHz}$ region) for the experiments. The FID was recorded for $40 \mu \mathrm{s}$, which generated a frequency resolution of $25 \mathrm{kHz}$ in our Fourier transformed microwave spectrum. The number of FIDs that were co-added to obtain the final spectra were 4.6 million in the case of pure linalool in the $2-8 \mathrm{GHz}$ range and 3 million for the rest of the spectra, that is, linalool in the 8$18 \mathrm{GHz}$ region and the two thyme oils in the $2-8 \mathrm{GHz}$ region.

The initial assignment of the observed experimental lines to rotational transitions of ${ }^{13} \mathrm{C}$ isotopologues of linalool and trans-thymol-A was performed through a fit based on an asymmetric rigid rotor Hamiltonian, using the $\mathrm{JB}^{34} 5^{34}$ and $\mathrm{PGOPHER}^{35}$ program packages. Refined fits were obtained using a standard Watson-type Hamiltonian (A-reduction and $\mathrm{I}^{\mathrm{r}}$ representation) by using the nonlinear least-squares fit program SPFIT developed by Pickett. ${ }^{36}$ The XIAM program was used to analyze the internal dynamics of linalool (conformer I) ${ }^{37}$ XIAM is a least squares fitting program employing the combined axis method of Woods, ${ }^{38,39}$ which is used to analyze the internal rotation features of molecules containing up to three internal rotors.

\subsection{Computational}

The conformational landscape of linalool was explored performing quantum-chemical calculations. Its initial minima were found with a conformational search by means of molecular mechanics with the SYBYL force field ${ }^{40}$ and using the SPARTAN program. ${ }^{41}$ When a suitable energy threshold was accomplished $(20 \mathrm{~kJ} / \mathrm{mol})$, the sixty most stable conformers were optimized using the B3LYPD3 dispersion-corrected density functional and the $6-311++\mathrm{G}(\mathrm{d}, \mathrm{p})$ basis set. Moreover, the lowest non-redundant conformers (less than $10 \mathrm{~kJ} / \mathrm{mol}$ ) were selected for further optimization of the structures and the calculation of their relative energies using a second-order perturbation theory 
(MP2) calculation with the $6-311++\mathrm{G}(\mathrm{d}, \mathrm{p})$ basis set. All the energy values used herein at the B3LYP-D3/6-311++G(d,p) and MP2/6-311++G(d,p) levels of theory are zero point corrected relative energies. Note that the B3LYP-D3 and MP2 calculations were performed in the harmonic approximation. The Cartesian coordinates of the five lowest energy conformers of linalool calculated at the MP2/6-311++G(d,p) level of theory are included in the Supplementary Information.

We followed the nomenclature used in ref. 5 for the conformations of thymol and carvacrol and the one used in ref. 6 for 4-carvomenthenol. For thymol and carvacrol, A and B refer to two different conformers, which differ in the position of the isopropyl group with respect to the hydroxyl group. In the B conformers, the value of the H-C7-C5-C6 dihedral angle (see Fig. 1, ref. 5 ) is $180^{\circ}$, while in the A conformers the value of this dihedral angle is $0^{\circ}$. In the case of 4carvomenthenol, the three conformations (A, B and C) differ in the rotation of the isopropyl group around the $\mathrm{C}-\mathrm{C}$ bond connecting the isopropyl group to the cyclohexene ring. The three conformers of 4-carvomenthenol were optimized at the MP2/6-311++G(d,p) level of theory to predict their dipole moment components. The trans-thymol-A conformer was optimized at the B3LYP-D3/6$311++\mathrm{G}(\mathrm{d}, \mathrm{p})$ level of theory to compare its results with the ones obtained at the MP2/6$311++\mathrm{G}(\mathrm{d}, \mathrm{p})$ level. The input structures were the ones published in refs. 5 (trans-thymol-A) and 6 (4-carvomenthenol).

The theoretical calculations were carried out using two software packages: (1) Spartan $16^{41}$ for the molecular mechanics conformational search and (2) Gaussian09 ${ }^{42}$ for the structure reoptimization of the most stable conformations and prediction of their relative energies and dipole moment components.

\section{Results and Discussion}

\subsection{Rotational analysis of two thyme oils: identification and semi-quantitative analysis of the chemical species present}

The top traces of Figs. 1 and 2 show several sections of the experimental broadband rotational spectra of the two natural thyme oils with different chemotypes recorded in the 2-8 GHz range. Five (thyme I) and four (thyme II) different terpenes could be identified based on their reported rotational constants (Table 1), present as twelve (thyme I) and six (thyme II) conformers, respectively. This can be seen by direct comparison with the simulated rotational spectra using experimental rotational constants reported in previous studies (Table 1) displayed in different colors in the bottom traces. The outstanding agreement between the experimental spectrum and the simulations illustrates one of the particular strengths of rotational spectroscopy, which is its high frequency accuracy and reproducibility.

Already upon inspection, trans-thymol-A is identified as the dominant species in the thyme I spectrum. Other strong components are camphor, followed by trans-thymol-B, cis-thymol-A, and trans- and cis-carvacrol-B. All components identified in thyme I are depicted in Figure 1 . In thyme II, both 4-carvomenthenol-A and linalool are prevailing, followed by 4-carvomenthenol-C and -B as well as camphor. The dominating species in thyme I, trans-thymol-A, is only a minor component in thyme II.

Note that the broadband rotational spectrum of thyme II is generally weaker than the thyme I spectrum by about a factor of four, which could be due to differences in the valve performance, for example, which we used to transfer the molecular mixture into the spectrometer chamber (see experimental section). Absolute intensities can vary significantly with the experimental parameters between different measurements, while relative intensity patterns are generally more reproducible. 
Table 1. Experimental rotational constants and theoretical absolute values of the dipole moment components for the different species present in the two thyme oils according to the rotational analysis carried out in this work.

\begin{tabular}{|c|c|c|c|c|c|c|c|c|c|c|c|}
\hline Conformer & Thyme I & Thyme II & Ref. & Formula & $\mathrm{A} / \mathrm{MHz}$ & $\mathrm{B} / \mathrm{MHz}$ & $\mathrm{C} / \mathrm{MHz}$ & $\left|\mu_{\mathrm{a}}\right| / \mathrm{D}^{b}$ & $\left|\mu_{\mathrm{b}}\right| / \mathrm{D}^{b}$ & 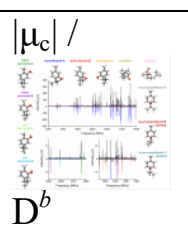 & $\begin{array}{l}\text { Boiling } \\
\text { point } \\
/{ }^{\circ} \mathrm{C}\end{array}$ \\
\hline trans-thymol-A & $\mathrm{X}^{a}$ & $\mathrm{X}$ & 5 & $\mathrm{C}_{10} \mathrm{H}_{14} \mathrm{O}$ & $2034.068(71)$ & $739.18202(35)$ & $591.51673(30)$ & 1.43 & 0.53 & 0.07 & 133 \\
\hline trans-thymol-B & $X$ & & 5 & $\mathrm{C}_{10} \mathrm{H}_{14} \mathrm{O}$ & $2261.070(62)$ & $708.92030(45)$ & $611.86246(50)$ & 1.50 & 0.72 & 0.04 & 133 \\
\hline cis-thymol-A & $X$ & & 5 & $\mathrm{C}_{10} \mathrm{H}_{14} \mathrm{O}$ & $2048.642(32)$ & $739.25463(50)$ & $590.31448(44)$ & 1.15 & 1.11 & 0.14 & 133 \\
\hline trans-carvacrol-B & $X$ & & 5 & $\mathrm{C}_{10} \mathrm{H}_{14} \mathrm{O}$ & $2159.0853(25)$ & $670.84871(51)$ & $577.52683(41)$ & 1.42 & 0.45 & 0.00 & 238 \\
\hline cis-carvacrol-A & $X$ & & 5 & $\mathrm{C}_{10} \mathrm{H}_{14} \mathrm{O}$ & $2274.0616(37)$ & $643.77212(46)$ & $564.76557(38)$ & 0.77 & 1.30 & 0.00 & 238 \\
\hline cis-carvacrol-B & $X$ & & 5 & $\mathrm{C}_{10} \mathrm{H}_{14} \mathrm{O}$ & $2152.8772(44)$ & $672.34073(50)$ & $578.22275(42)$ & 1.05 & 1.14 & 0.00 & 238 \\
\hline linalool & $X$ & $\mathrm{X}$ & 10 & $\mathrm{C}_{10} \mathrm{H}_{18} \mathrm{O}$ & $1646.73627(25)$ & $682.19766(15)$ & $618.75034(18)$ & 1.12 & 0.04 & 1.87 & 198 \\
\hline 4-carvomenthenol A & $X$ & $\mathrm{X}$ & 6 & $\mathrm{C}_{10} \mathrm{H}_{18} \mathrm{O}$ & $2370.3204(13)$ & $683.17421(30)$ & $606.39955(24)$ & 1.39 & 0.30 & 1.01 & 212 \\
\hline 4-carvomenthenol B & $X$ & $X$ & 6 & $\mathrm{C}_{10} \mathrm{H}_{18} \mathrm{O}$ & $2255.1848(23)$ & $673.70463(35)$ & $646.54551(32)$ & 1.23 & 0.82 & 0.91 & 212 \\
\hline 4-carvomenthenol C & $\mathrm{X}$ & $\mathrm{X}$ & 6 & $\mathrm{C}_{10} \mathrm{H}_{18} \mathrm{O}$ & $2236.4047(12)$ & $674.83599(26)$ & $647.30427(19)$ & 1.15 & 0.29 & 1.20 & 212 \\
\hline & & & & & & & & 2.96 & 0.68 & 0.09 & $\rho \rho 0$ \\
\hline
\end{tabular}

${ }^{a}$ The components marked with an "X" are present in the corresponding essential oil according to the rotational analysis of this work.

${ }^{b}$ The dipole moment components of the species were calculated at the MP2/6-311++G(d,p) level of theory.

${ }^{c}$ The experimental dipole moment components of camphor are determined from Stark effect measurements. ${ }^{15}$ 


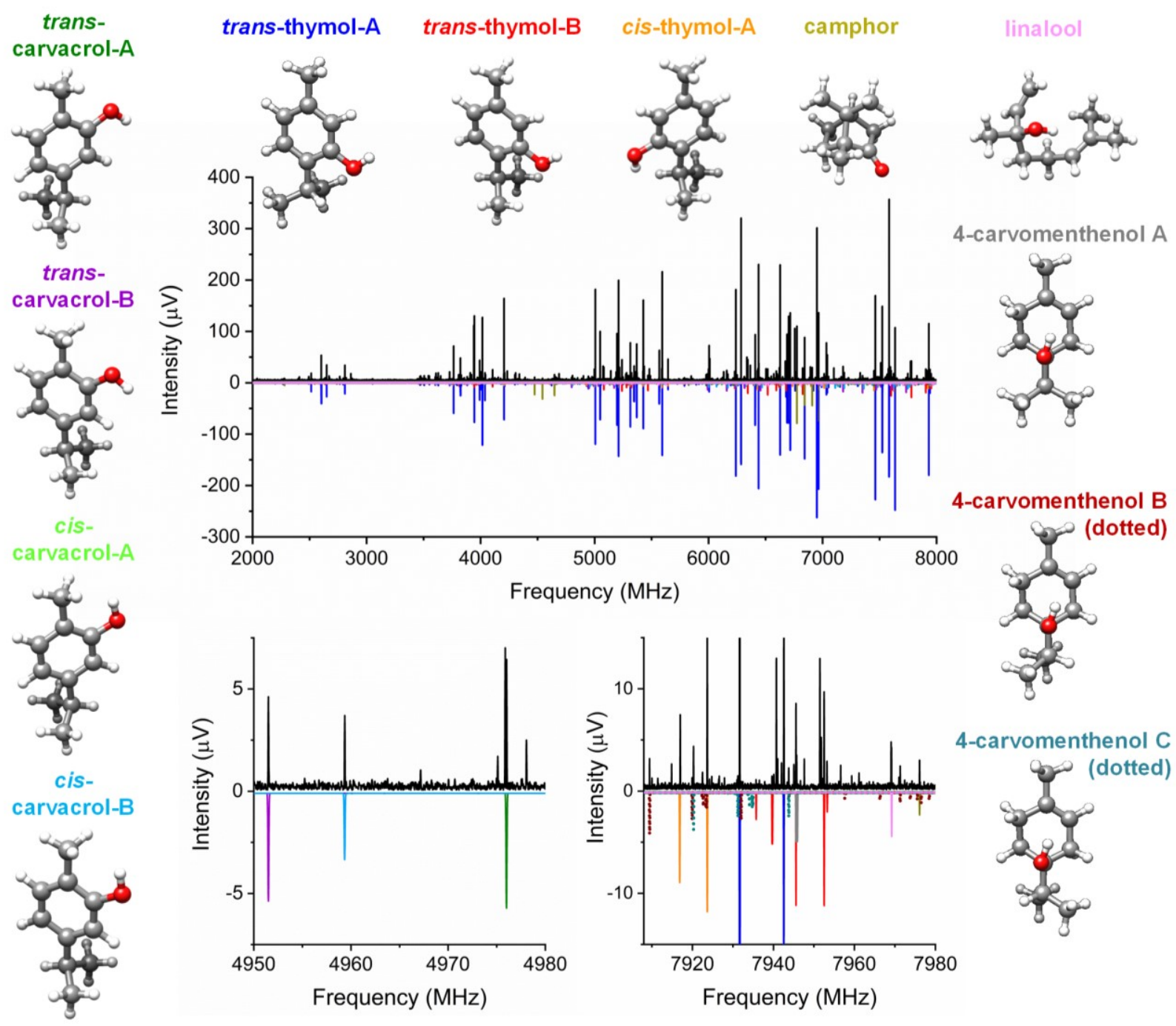

Figure 1. Overview and zoom-ins of the broadband rotational spectrum of thyme I. The upper traces depict the experimental spectrum (black color), and the lower traces show the simulated rotational spectra of the different terpenes and their conformers (in different colors) identified in the oil, based on their reported experimental rotational constants (Table 1). The structures of the different conformers are optimized at the MP2/6-311++G(d,p) level of theory. 


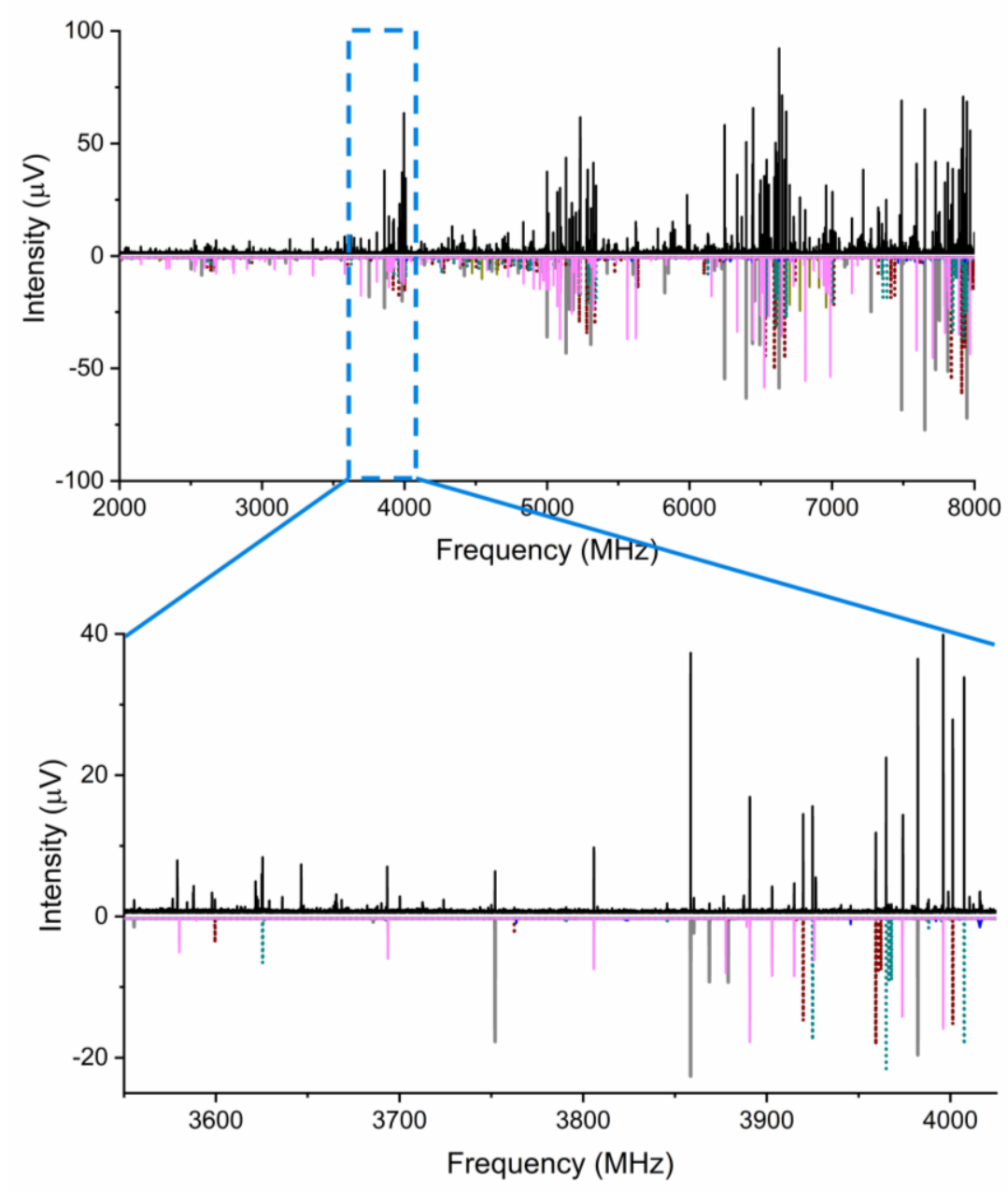

Figure 2. Experimental (black color) and simulated (different colors, see Fig. 1 for conformer labeling) broadband rotational spectra of thyme II essential oil in the broadband $2000-8000 \mathrm{MHz}$ and, as a zoom-in, in the 3550-4025 MHz spectral regions.

In the weak pulse limit of CP-FTMW spectroscopy, the observed transition strength depends on the number of molecules in the sample, the square of the transition dipole moments, the electric field strength, and the population difference between the respective rotational states at equilibrium. ${ }^{43,44}$ The vapor pressures of the different terpenes of the oil are increased by heating before they are supersonically expanded. These vapor pressures are related to the respective boiling points (Table 1) and are mixture-dependent in such a multi-component sample. This can result in somewhat different number densities of the constituents in the molecular jet compared to the liquid oil. Note that during the course of the experiment, these number densities can also vary, because molecules with higher vapor pressures might be depleted sooner than others with lower vapor pressures. We approached this by comparing the spectra recorded at different temperature conditions $\left(80{ }^{\circ} \mathrm{C}, 90^{\circ} \mathrm{C}\right.$ and $120^{\circ} \mathrm{C}$ for thyme I oil and $80{ }^{\circ} \mathrm{C}, 90^{\circ} \mathrm{C}$ and $100{ }^{\circ} \mathrm{C}$ for thyme II oil) and observed only minor changes. Table S1 in the Supplementary Information summarizes the vapor pressures of the terpenes we identified at different temperatures. The vapor pressures change in a similar way for the terpenes for the different temperatures (see values marked in red in Table $\mathrm{S} 1$ ). Thus, the composition of the vapor at different temperatures can be assumed to be comparable, in agreement with our measurements at different temperatures. Table S2 shows the vapor pressures of the respective terpenes at $25{ }^{\circ} \mathrm{C}$. They are comparable apart from camphor, which has a larger vapor pressure. Thus, we can assume that the compositions of the oils at $25^{\circ} \mathrm{C}$ may consist of a larger amount of camphor than the compositions at $80-120^{\circ} \mathrm{C}$, where camphor might be depleted. 
The abundances of the other terpenes should be comparable at $25{ }^{\circ} \mathrm{C}$ and at $80-120{ }^{\circ} \mathrm{C}$. In addition, we performed large numbers of averages to counteract fluctuations in the evaporation process. Moreover, we have to take into account the frequency dependence of the TWT amplifier and other frequency-dependent components, such as the horn antennas used to broadcast the microwave radiation into the interaction zone of the spectrometer, on the overall excitation intensity and thus on the transition intensities. In principle, this effect and further frequency-dependent components can be accounted for with an instrument function, which was not performed as part of this study. Instead, we focused our analysis on a rather narrow frequency range (see below) where the frequency dependence should be negligible. Because of the previous factors, determining the abundances of the different species present in a complex mixture, such as an essential oil, based on the intensities of rotational transitions can be a challenging task. The qualitative assignment and species identification, however, solely relies on the characteristic, precise, and highly reproducible high-resolution rotational transition frequencies.

In this work, we have estimated the abundances of the constituents of the oils in a semiquantitative way from intensity measurements of selected transitions in the 5-6 GHz region, where we expect a flat frequency response of our spectrometer, and focused on a small selection of a-type rotational transitions $\left(J_{K a K c}: 4_{14}-3_{13}, 4_{04}-3_{03}\right.$ and $\left.4_{13}-3_{12}\right)$. All components of the oils under consideration have a suitable value of the $a$ dipole moment component $\left(\mu_{\mathrm{a}}\right)$ (Table 1$)$. Note that several conformers (three conformers of thymol, two conformers of trans-carvacrol and the one linalool conformer) in these oils present methyl group internal rotation, resulting in line splittings into two or more components, so that the sum of the intensities needs to be taken into account.

To be able to compare the intensities of the different species to get an indication of their abundance in the oil, we divided the intensities of the $a$-type transitions $4_{14}-3_{13}, 4_{04}-3_{03}$ and $4_{13}-3_{12}$ (if available, see Table S3) of each conformer by the square of their respective $\mu_{\mathrm{a}}$ value calculated at the MP2/6-311++G(d,p) level of theory (see Table 1). The population differences between the rotational states involved in the respective transitions are calculated to be similar for rotational temperatures around $1.5 \mathrm{~K}$, so that their influence can be assumed to be negligible. The resulting values were normalized with respect to the most abundant conformers, that is, trans-thymol-A in thyme I and linalool in thyme II. The three normalized $\mathrm{I} / \mu_{\mathrm{a}}{ }^{2}$ values (one for each transition of the above-mentioned series) obtained for each conformer were averaged (see Table S5). Following this procedure, the estimated abundances of the different conformers in thyme I are trans-thymol-A $>$ cis-thymol-A $>$ trans-thymol-B $>$ cis-carvacrol-A $>$ camphor $\approx$ trans-carvacrol-A $>$ linalool $\approx$ cis carvacrol-B $\approx$ trans-carvacrol-B $>4$-carvomenthenol $\mathrm{A} \approx 4$-carvomenthenol $\mathrm{B} \approx 4$-carvomenthenol $\mathrm{C}$ (see Table 2.a).

To compare our estimated abundances with those provided by the supplier, Florihana, we added the values for the different conformers present for each terpene, obtaining the following estimated order in abundances in thyme $\mathbf{I}$ according to thymol $>$ carvacrol $>$ camphor $>$ linalool $\approx$ 4-carvomenthenol (see Table 2.b). This result agrees with the gas chromatography analyses provided by the supplier (see Table 2.b and Table S6), apart from linalool, which is present as 13$16 \%$ based on their analyses but only as a minor component in our study. This discrepancy could have a number of reasons: a) Linalool shows extensive line splitting due to methyl group internal rotation, as discussed in section 3.3. Even though carefully considered, this might have an influence on the analysis of the intensities. b) Generally, the population of a flexible molecule can be distributed over several conformers, as in the case of carvacrol or 4-carvomenthenol. As discussed below, the next linalool conformer is about $5 \mathrm{~kJ} / \mathrm{mol}$ higher in energy, for which we would expect only low population under the cold conditions of a molecular jet, so that they cannot be detected with microwave spectroscopy. However, a non-zero population could falsify the abundance estimates and result in somewhat lower values for the observed conformer. c) Essential oils are natural products with varying composition. As mentioned in section 2.1. Experimental, the supplier did not provide the specific gas chromatography analyses of the samples we analysed, but the ones of several badges for these two chemotypes of oils in general. ${ }^{31}$ Smaller variations in the composition are thus possible. 
Table 2. a) Percentages calculated from the averaged normalized $I / \mu_{\mathrm{a}}{ }^{2}$ values given in Table $\mathrm{S} 5$ for thyme I oil. b) Ratios of the different terpenes calculated from these percentages (\%) and normalized with respect to thymol and the ones obtained from the supplier Florihana for the different terpenes identified in thyme I oil.

\begin{tabular}{|c|c|c|c|c|}
\hline \multicolumn{5}{|c|}{ THYME I } \\
\hline a) Conformer & $\begin{array}{c}\text { Normalized } \mathrm{I} / \boldsymbol{\mu}_{\mathrm{a}}{ }^{2} \\
\text { values } \pm \text { Error }^{a}\end{array}$ & \multicolumn{3}{|c|}{$\begin{array}{c}\% \text { Normalized } I / \mu_{\mathrm{a}}{ }^{2} \\
\text { values }\end{array}$} \\
\hline trans-thymol-A & $1.00 \pm 0.20^{b}(0.02)^{c}$ & \multicolumn{3}{|c|}{61.7} \\
\hline trans-thymol-B & $0.12 \pm 0.02(0.004)$ & \multicolumn{3}{|c|}{7.4} \\
\hline cis-thymol-A & $0.19 \pm 0.04(0.007)$ & \multicolumn{3}{|c|}{11.7} \\
\hline trans-carvacrol-A & $0.05 \pm 0.01(0.005)$ & \multicolumn{3}{|c|}{3.1} \\
\hline trans-carvacrol-B & $0.03 \pm 0.007(0.004)$ & \multicolumn{3}{|c|}{1.9} \\
\hline cis-carvacrol-A & $0.07 \pm 0.02(0.013)$ & \multicolumn{3}{|c|}{4.3} \\
\hline cis-carvacrol-B & $0.04 \pm 0.01(0.007)$ & \multicolumn{3}{|c|}{2.5} \\
\hline linalool & $0.03 \pm 0.01(0.006)$ & \multicolumn{3}{|c|}{1.9} \\
\hline 4-carvomenthenol A & $0.008 \pm 0.004(0.004)$ & \multicolumn{3}{|c|}{0.6} \\
\hline 4-carvomenthenol B & $0.007 \pm 0.005(0.005)$ & \multicolumn{3}{|c|}{0.6} \\
\hline 4-carvomenthenol C & $0.02 \pm 0.006(0.006)$ & \multicolumn{3}{|c|}{1.2} \\
\hline camphor & $0.05 \pm 0.006$ & \multicolumn{3}{|c|}{3.1} \\
\hline \multirow[t]{2}{*}{ b) Terpenes } & \multirow{2}{*}{$\begin{array}{c}\% \text { Normalized } \mathrm{I} / \boldsymbol{\mu}_{\mathrm{a}}{ }^{2} \\
\text { values }\end{array}$} & \multicolumn{3}{|c|}{ Ratios } \\
\hline & & $\begin{array}{c}\text { Our } \\
\text { work }\end{array}$ & & lier $^{d}$ \\
\hline thymol & 80.9 & 1.00 & 1.00 & 1.00 \\
\hline carvacrol & 11.7 & 0.15 & 0.10 & 0.09 \\
\hline linalool & 1.9 & 0.02 & 0.16 & 0.13 \\
\hline 4-carvomenthenol & 2.5 & 0.03 & 0.03 & $<0.30^{e}$ \\
\hline camphor & 3.1 & 0.04 & 0.07 & $<0.30^{e}$ \\
\hline
\end{tabular}

${ }^{a}$ Error calculated according to the propagation error formulas $f=\frac{(x \pm \Delta x)}{(y \pm \Delta y)}=\left(\frac{x}{y}\right) \pm\left(\frac{x}{y}\right) \sqrt{\left(\frac{\Delta x}{x}\right)^{2}+\left(\frac{\Delta y}{y}\right)^{2}}$ and $f=(z \pm \Delta z)^{2}=z^{2} \pm z^{2}\left(\frac{2 \Delta z}{z}\right)$ (see Table S4 ESI for detailed explanation).

${ }^{b}$ Errors calculated considering a $10 \%$ error in the calculation for $\mu_{\mathrm{a}}$.

${ }^{c}$ Errors in parentheses and in Italic font are calculated considering a $1 \%$ error in the calculations for $\mu_{\mathrm{a}}$.

${ }^{d}$ Ratios obtained from gas chromatography analyses of the lot numbers B221217ES (left) and L121018ES (right) available on the supplier webpage. ${ }^{31}$

$e$ Percentages not specified by the supplier. They provide $87.9 \%$ of the components for lot number L121018ES. Thus, in the case that 4-carvomenthenol and camphor could be present they would be in a significantly lower amount than $12.1 \%$, that is, a ratio lower than 0.30 (see Table S6 ESI). ${ }^{31}$

In Table 2.a, we provide the errors calculated for our normalized $I / \mu_{\mathrm{a}}{ }^{2}$ values based on the general error propagation formula (see ESI for a detailed explanation of the error calculations). We consider the error in the prediction of the theoretical (MP2/6-311++G(d,p)) $\mu_{\mathrm{a}}\left(\Delta \mu_{\mathrm{a}}\right)$ to be $10 \%{ }^{45,46}$ In the case of camphor, we use the experimental $\mu_{\mathrm{a}}$ value determined from Stark effect measurements and its corresponding error (2.9934 (23) D). ${ }^{15}$ The difference between the calculated (Table 1) and experimental $\mu_{\mathrm{a}}$ value for camphor is only about $1 \%$. However, for other types of molecules deviations on the order of 6-9 \% have been reported. ${ }^{45}$ Thus, errors in the calculated dipole moment of up to $10 \%$ are a conservative assumption. The errors obtained for the normalized $\mathrm{I} / \mu_{\mathrm{a}}{ }^{2}$ ratios of the different conformers are around $20 \%$ because of the estimated uncertainty in the calculated dipole moment components of $10 \%$. Errors of experimental dipole moments determined using Stark effect measurements are typically below $1 \%,{ }^{47}$ resulting in much smaller errors for the $\mathrm{I} / \mu_{\mathrm{a}}{ }^{2}$ ratios values, as can be seen in the case of camphor. The errors increase for the weaker terpenes, because we are considering the error in our intensity values to be $1 \mu \mathrm{V}$ (twice the noise 
level), and the intensities of the transitions for these weak terpenes are lower than $9 \mu \mathrm{V}$ (see Table S3 ESI).

Table 3. a) Percentages calculated from the averaged normalized $\mathrm{I} / \mu_{\mathrm{a}}{ }^{2}$ values obtained in Table S5 for thyme II oil. b) Ratios of the different terpenes calculated from these percentages (\%) and normalized with respect to linalool and the ones obtained from the supplier Florihana for the different terpenes identified in thyme II oil.

\begin{tabular}{|c|c|c|c|c|c|}
\hline \multicolumn{6}{|c|}{ THYME II } \\
\hline a) Conformer & 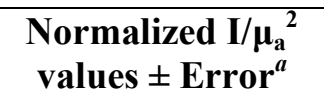 & \multicolumn{4}{|c|}{$\%$ Normalized $\mathrm{I} / \boldsymbol{\mu}_{\mathrm{a}}{ }^{2}$ values } \\
\hline linalool & $1.00 \pm 0.20^{b}(0.03)^{c}$ & \multicolumn{4}{|c|}{40.5} \\
\hline trans-thymol-A & $0.04 \pm 0.01(0.01)$ & \multicolumn{4}{|c|}{1.6} \\
\hline camphor & $0.04 \pm 0.02$ & \multicolumn{4}{|c|}{1.6} \\
\hline 4-carvomenthenol A & $0.40 \pm 0.08(0.01)$ & \multicolumn{4}{|c|}{16.2} \\
\hline 4-carvomenthenol B & $0.26 \pm 0.06(0.02)$ & \multicolumn{4}{|c|}{10.5} \\
\hline 4-carvomenthenol C & $0.73 \pm 0.15(0.02)$ & \multicolumn{4}{|c|}{29.6} \\
\hline \multirow[t]{2}{*}{ b) Terpenes } & \multirow{2}{*}{$\begin{array}{c}\text { \% Normalized } \\
\mathbf{I} / \mu_{\mathrm{a}}{ }^{2} \text { values }\end{array}$} & \multicolumn{4}{|c|}{ Ratios } \\
\hline & & $\begin{array}{c}\text { Our } \\
\text { work }\end{array}$ & \multicolumn{3}{|c|}{ Supplier $^{d}$} \\
\hline linalool & 40.5 & 1.00 & 1.00 & 1.00 & 1.00 \\
\hline trans-thymol-A & 1.6 & 0.04 & 0.01 & 0.02 & 0.01 \\
\hline camphor & 1.6 & 0.04 & 0.03 & $<0.28^{e}$ & 0.01 \\
\hline 4-carvomenthenol & 56.3 & 1.39 & 0.46 & 0.44 & 0.19 \\
\hline
\end{tabular}

${ }^{a}$ Error calculated according to the propagation error formulas $f=\frac{(x \pm \Delta x)}{(y \pm \Delta y)}=\left(\frac{x}{y}\right) \pm\left(\frac{x}{y}\right) \sqrt{\left(\frac{\Delta x}{x}\right)^{2}+\left(\frac{\Delta y}{y}\right)^{2}}$ and $f=(z \pm \Delta z)^{2}=z^{2} \pm z^{2}\left(\frac{2 \Delta z}{z}\right)$ (see Table S4 ESI for detailed explanation).

${ }^{b}$ Errors calculated considering a $10 \%$ error in the calculation for $\mu_{\mathrm{a}}$.

${ }^{c}$ Errors in parentheses and in Italic font are calculated considering a 1\% error in the calculations for $\mu_{\mathrm{a}}$.

${ }^{d}$ Ratios obtained from gas chromatography analyses of the lot numbers B280917ES (left), L280818ES (middle) and B230419ES (right) available on the supplier webpage. ${ }^{31}$

${ }^{e}$ Percentage not specified by the supplier. They provide $90.8 \%$ of the components for lot number L280818ES. Thus, in the case that camphor could be present it would be in a significantly lower amount than $9.2 \%$, that is, a ratio lower than 0.28 (see Table S6 ESI). ${ }^{31}$

Following the same procedure for thyme II, the estimated abundances for the different conformers are linalool $>4$-carvomenthenol $\mathrm{C}>4$-carvomenthenol $\mathrm{A}>4$-carvomenthenol $\mathrm{B}>$ camphor $\approx$ trans-thymol-A (see Table 3.a). After averaging over all the conformers and normalizing with respect to linalool, which is the most abundant terpene according to the supplier, we obtain the following order of abundances for the terpenes in thyme II: 4-carvomenthenol $>$ linalool $>$ camphor $\approx$ trans-thymol-A (see Table 3.b). Interestingly, when taking all conformers together, we observe that 4-carvomenthenol is more abundant than linalool, in contrast to the supplier information. Thus, again, we find linalool to be in lower abundance compared to the information provided by the supplier. As discussed above, this can have different reasons. While the GC results from the supplier are only considering specific samples (it can be observed how the 4carvomenthenol ratio changes for one of the supplier badges), our analysis also has certain weaknesses. We need to make sure that the abundances in the gas phase represent those in the liquid oil (a challenge that we share with GC). As mentioned, we approached this by acquiring a large number of acquisitions (up to 3 million) and measurement times of 15 hours. We also varied the temperature of the sample used for increasing the vapor pressure to investigate its influence on the sample composition observed with rotational spectroscopy and found it to be minor. This is in accordance with the vapor pressures for the different terpenes (Table S1), since they vary in a similar manner with temperature. The most critical aspect might be the rather small number of 
rotational transitions considered for the analysis as well as the complication due to internal rotation line splittings.

In the gas chromatography analyses, more terpenes were recorded than we include in our study. While rotational spectroscopy is powerful and allows for unambiguous identification of molecules once their rotational constants are known, it can be difficult to determine which molecule is observed without prior knowledge, i.e., information on the molecular mass or atomic composition, for example. As a consequence, we restricted ourselves to the stronger components ( $>$ $5 \%$ ) of the oils for which microwave spectroscopy information was already available. In such a complex mixture, it will be challenging (but not impossible), to identify weaker components based on quantum-chemical rotational constants, which often give deviations of several tens and even hundreds $\mathrm{MHz}$ of the predicted rotational transitions from the experimental ones.

If we only consider the components that are present in the samples with an amount larger than $5 \%$, ${ }^{31}$ myrcene, $\alpha$-terpinene, and $\gamma$-terpinene should have also been observed in thyme II oil, where they are present with percentages around 3-9\%, and para-cymene and $\gamma$-terpinene in thyme I oil, where their respective percentages are 18-21\% and 11-13\%. Para-cymene, $\alpha$ - and $\gamma$-terpinene have small dipole moments $(0.1 \mathrm{D}$ for para-cymene and less than $0.1 \mathrm{D}$ for $\alpha$ - and $\gamma$-terpinene according to quantum-chemical calculations) and are thus difficult to be observed by rotational spectroscopy in these mixtures. Myrcene should in principle be observable because its theoretical dipole moment is on the order of $0.4 \mathrm{D}$. However, it could be that the actual amount of myrcene is lower than $5-8 \%$ in our sample, and that the calculated rotational constants from quantum-chemical calculations are not sufficient to assign these rather low-concentration/low-dipole moment species directly in the oil.

In general, the percentages we use in the comparison (Tables 2, 3, and S6) were normalized with respect to the most abundant terpenes thymol (thyme I) and linalool (thyme II). So we are not giving overall percentages.

In summary, in agreement with the supplier information, we obtained remarkably different compositions for the two thyme oils thyme I and thyme II, with the most abundant species in thyme I being the least abundant one in thyme II, and vice versa. We would like to emphasize here again that the assignment of the different species in the oils (including their conformers) is based on their remarkably different rotational spectra. This makes rotational spectroscopy a unique tool to qualitatively analyze this kind of complex molecular mixtures, which is not fully explored for analytic purposes yet. A main error contribution in the abundance analysis for a more quantitative insight arises from the uncertainty in calculated dipole moments. The use of experimental dipole moments, if available, can reduce the errors significantly, as in the case of camphor. A particular strength is that also precise structural information can be obtained from the rotational spectra, as presented in the next section for the cases of trans-thymol-A and linalool, for which no experimental structures have been reported before.

\subsection{Substitution structures for trans-thymol-A and linalool}

The signal-to-noise ratio of the most abundant conformer trans-thymol-A in thyme I essential oil was clearly sufficient $(600: 1)$ to observe the rotational spectra of all singly substituted ${ }^{13} \mathrm{C}$ isotopologues in natural abundance $(1.1 \%)$. With a SNR of about 120:1, this is not the case for linalool in thyme II. To determine the structure of linalool, we thus recorded the broadband rotational spectrum of pure linalool, giving us access to all the singly substituted ${ }^{13} \mathrm{C}$ species and to allow for a more detailed analysis of the internal rotation (see below). The experimentally determined rotational constants of all ${ }^{13} \mathrm{C}$ isotopologues for both molecules are summarized in Tables S7 and S8 in the Supporting Information. We could obtain their full heavy-atom substitution $\left(r_{s}\right)$ structures through the Kraitchman equations using the KRA program, ${ }^{20,48,49}$ by determining the atom coordinates in the principal axis system from the isotopic changes in the moments of inertia. This method exploits mono-isotopic substitution information. It presents limitations when the atom is located in plane or near the principal axes, and it only provides the magnitudes of the atom 
coordinates. The respective signs can be taken from a quantum-chemical calculation or using chemical intuition. For trans-thymol-A, C5 and C7 are located near the principal axes and in the $a b$ plane. In the case of linalool, $\mathrm{C} 10$ is in the $a b$ plane and $\mathrm{C} 7$ is on the $a$ principal axis. For these carbon atoms, imaginary values of the coordinates were obtained after applying Kraitchman's equations (see Tables S9 and S10, Supporting Information). The values of these coordinates were set to zero to determine the structural parameters. Despite these limitations, the calculated (MP2/6$311++\mathrm{G}(\mathrm{d}, \mathrm{p}))$ and the experimental $\left(\mathrm{r}_{\mathrm{s}}\right)$ positions for these carbon atoms are in a good agreement.

Improved structural information can be obtained with a least-squares fitting of certain distances and angles, which allows exploiting multi-isotopic information. It reproduces all the experimental moments of inertia in a vibrational state. The simplest type of such a fit involves fitting the structural parameters of the effective ground state, $r_{0}$. The effective structures $\left(r_{0}\right)$ for trans-thymol-A and linalool were obtained through least-squares fits of the ${ }^{13} \mathrm{C}$ experimental moments of inertia using the STRFIT program. ${ }^{48}$ We could determine the internal coordinates involving these atoms, that is, 16 and 18 parameters for trans-thymol-A and linalool, respectively. In Fig. 3, a comparison between the experimental $r_{0}$ and $r_{s}$ structures is displayed, showing a good agreement. The underlying structure (grey for carbons, red for oxygen) is the one obtained from the $r_{0}$ fit, while the determined $r_{s}$ atom positions are shown as blue spheres. The coordinates are summarized in Tables S9 and S10. The experimental structural $r_{0}$ and $r_{s}$ parameters are given in Tables S11 and S12, in comparison with the $r_{e}$ equilibrium ones (MP2/6-311++G(d,p) and B3LYP$\mathrm{D} 3 / 6-311++\mathrm{G}(\mathrm{d}, \mathrm{p}))$. Both theoretical levels reproduce the substitution structures in a suitable way.
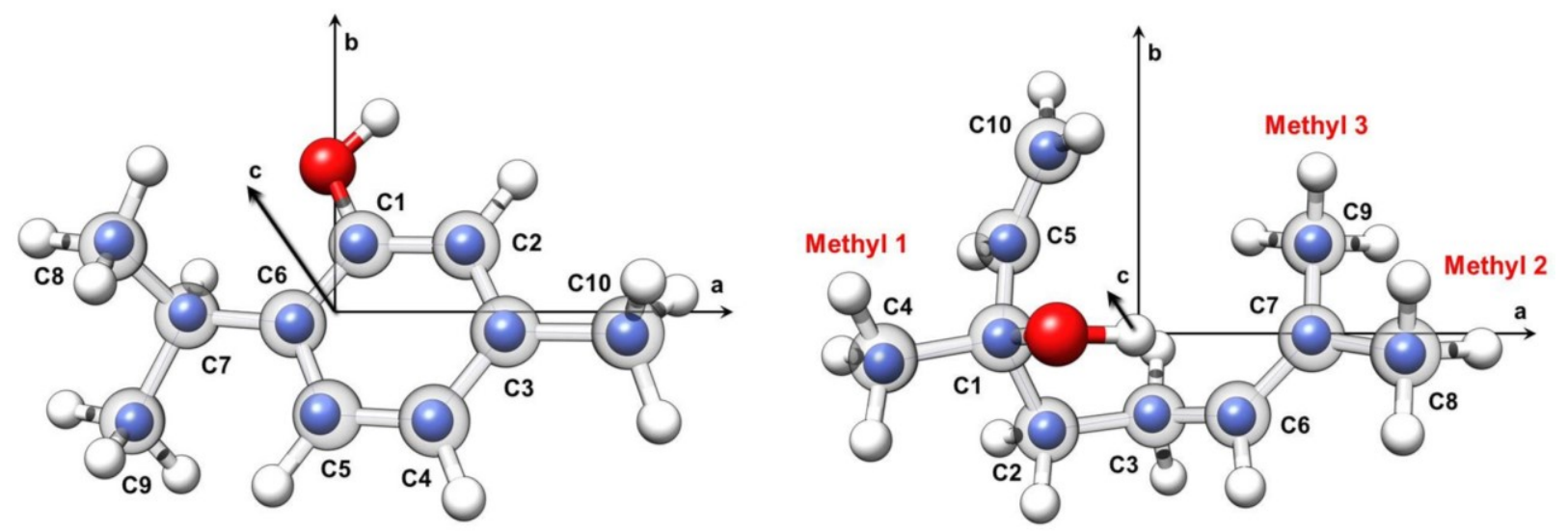

Figure 3. Comparison of the experimentally determined $r_{s}$ (blue spheres) and $r_{0}$ (grey atoms) structures for trans-thymol-A (left) and linalool (right).

\subsection{Conformational analysis and internal rotation of linalool}

The conformational landscape of linalool was studied previously by Nguyen et al. ${ }^{10}$ using a combination of quantum-chemical calculations and cavity-based molecular-beam Fourier transform microwave (MB-FTMW) spectroscopy in the 9-16 GHz region. They identified one conformer of this terpene in the gas phase, which exhibits internal rotation of one methyl top. The second conformer in energy was calculated to be about $5 \mathrm{~kJ} / \mathrm{mol}$ higher and could not be determined experimentally due to its low population. ${ }^{10}$ Linalool is a flexible and linear molecule; for this reason, it could be expected to present more than one conformer in the supersonic expansion, as in the case of citronellal, another linear terpene, for which 15 conformers were identified in a recent microwave spectroscopy study. ${ }^{50}$ For this reason, we revisited linalool's conformational preference by performing a conformational search using molecular mechanics followed by a structure reoptimization of its conformers and prediction of their relative energies using B3LYP-D3 and MP2 calculations (see section 2.2. Computational). For the lowest-energy conformers, the theoretical results were very similar to the ones obtained by Nguyen et al., ${ }^{10}$ finding conformer I and conformer 
II as the most stable ones with respective relative energies of 0.0 and $5.3 \mathrm{~kJ} / \mathrm{mol}$. The conformational searches of the two studies rendered some different conformations for higher energy conformers $(>7 \mathrm{~kJ} / \mathrm{mol})$. The fact that there are no additional conformations below $5 \mathrm{~kJ} / \mathrm{mol}$ is striking because of linalool's linear structure.

Linalool (conformer I) has three methyl tops (Fig. 3), labeled as 1 (containing C4), 2 (containing C8) and 3 (containing C9), with calculated internal rotation barriers (MP2/6$311++\mathrm{G}(\mathrm{d}, \mathrm{p})$ level of theory) of $15.9 \mathrm{~kJ} / \mathrm{mol}\left(1327 \mathrm{~cm}^{-1}\right), 3.9 \mathrm{~kJ} / \mathrm{mol}\left(324 \mathrm{~cm}^{-1}\right)$, and $9.3 \mathrm{~kJ} / \mathrm{mol}$ $\left(776 \mathrm{~cm}^{-1}\right)$, respectively. ${ }^{10}$ The calculated barriers for methyl tops 2 and 3 are lower, as expected, since they are attached to the same $\mathrm{sp}^{2}$ hybridized carbon atom $\mathrm{C} 7$. Note that their barriers differ since they are surrounded by different chemical environments. Nguyen et al. assigned the observed internal rotation splitting pattern into so-called A and E states to the internal rotation of methyl group 2 and determined a barrier of $4.7876(77) \mathrm{kJ} / \mathrm{mol}\left[400.20(64) \mathrm{cm}^{-1}\right]$. Furthermore, they reported the observation of some multiplets with components spaced by $10 \mathrm{kHz}$, as well as broadened lines for other transitions, which were not considered in their analysis.

In our broadband rotational spectrum of pure linalool, which we recorded to observe the rotational fingerprints of the ${ }^{13} \mathrm{C}$ isotopologue to determine linalool's carbon backbone structure (vide supra), we observed a number of rotational transitions split into multiplets of up to five rotational lines with components spanning around 50-200 MHz (Figure 4). These multiplets belong to linalool (conformer I) and clearly indicate a complex internal dynamics arising from more than one methyl top. Due to the broadband capabilities of our spectrometer a large frequency range of the spectrum could be recorded and then we could analyze and fit these lines using the program XIAM, considering intramolecular dynamics arising from two methyl groups. The observed splitting pattern into five components due to the two methyl groups can be described as follows. Starting with the lower barrier, methyl 2 splits the unperturbed transition into A and E components. These two components are further split by the internal motion of methyl 3, leading to four components $\mathrm{AA}, \mathrm{AE}, \mathrm{EA}$, and $\mathrm{EE}$. The EE component is further split into $\mathrm{EE}^{+}$and $\mathrm{EE}^{-}$due to toptop interactions. ${ }^{16}$ Note that this last splitting is only resolved for $c$ type transitions with $\mathrm{K}_{\mathrm{a}}=3$.

The starting point of the analysis was a subset with $83 a$-and $c$-type lines, corresponding to the AA internal rotation components, that was fit to a rigid-rotor Hamiltonian with a standard deviation of $19.7 \mathrm{kHz}$ (see Table S13). We then used this fit together with the experimental internal rotation barrier determined previously for methyl $2^{\text {ref.10 }}$ and the theoretical one for methyl 3 to predict the internal rotation splittings due to the two methyl tops. Using XIAM, 230 rotational transitions were then fit to a standard deviation of $9.6 \mathrm{kHz}$, determining the rotational and centrifugal distortion constants as well as the internal rotation barriers for methyl tops 2 and 3 . The experimental intensities of our transitions agree with the corresponding intensities from the spin statistical weights, ${ }^{51,52}$ with an exception for AA, which is partly overlapping with AE. The angles $\delta$ and $\varepsilon$ are used to specify the orientation of the methyl rotors within the principal axes of inertia, and both $\delta$ and $\varepsilon$ could be determined for methyl 2, whereas none of them could be determined in the case of methyl 3. Methyl 1 is attached to the chiral $\mathrm{sp}^{3}$ hybridized carbon $\mathrm{C} 1$ of the molecule together with a hydroxyl group, a vinyl group and an alkyl chain, resulting in a rather high barrier. $^{10,16}$ The corresponding splittings are thus too small to be resolved using the COMPACT spectrometer, where the resolution is approximately $25 \mathrm{kHz}$. 

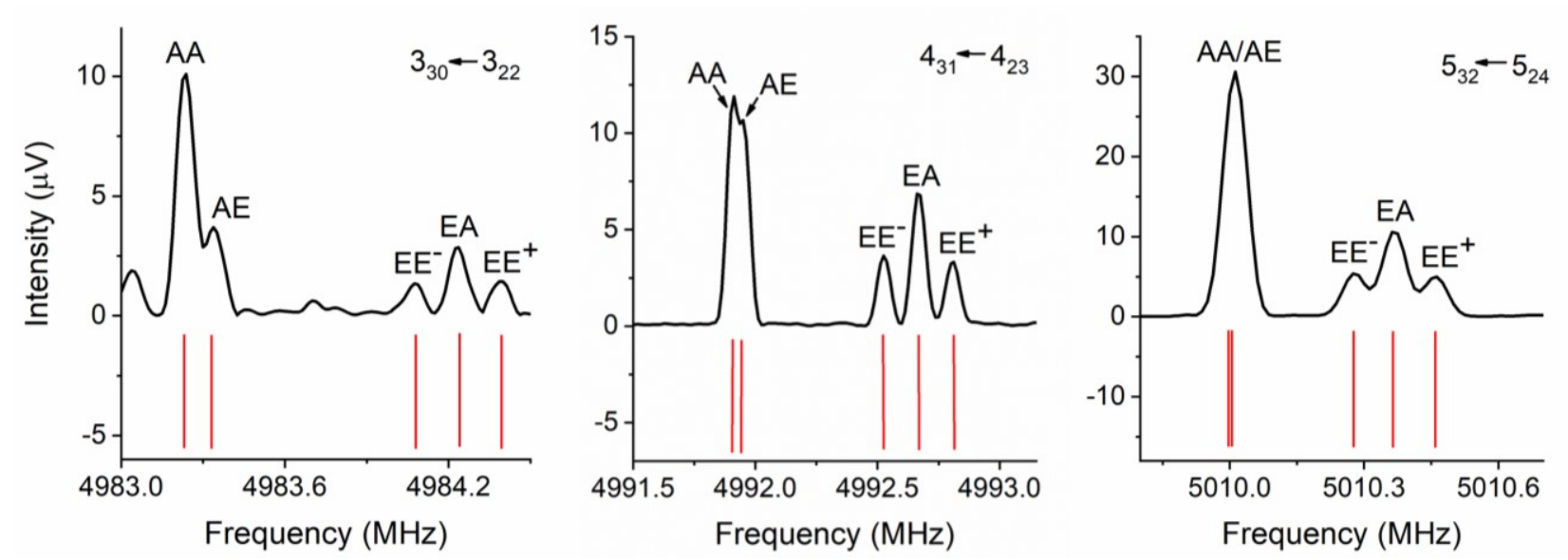

Figure 4. Three sections of the broadband rotational spectrum of linalool (conformer I) illustrating the multiplet structure due to internal rotation. The upper traces (black) show the experimental spectrum, while the lower traces (red) are stick spectra based on simulations of the fitted rotational parameters using XIAM.

In order to include more lines into our fit, the $8-18 \mathrm{GHz}$ frequency range was also recorded using the COMPACT spectrometer. Finally, 384 lines were assigned and fit with a standard deviation of $10.8 \mathrm{kHz}$ in the 2-18 GHz range (see Tables $\mathrm{S} 14$ and $\mathrm{S} 15$, which contain a comparison with the results obtained by Nguyen et al. ${ }^{10}$ and a complete list collecting all the observed transitions, respectively). The experimentally determined values for the internal rotation barriers for methyl 2 and 3 are 4.7703(96) $\mathrm{kJ} / \mathrm{mol}$ and 9.2581(74) $\mathrm{kJ} / \mathrm{mol}$, respectively. Due to the globular linalool structure, methyl 3 is located close to the alkyl chain, especially to one of the $-\mathrm{CH}_{2}-$ groups (ca. $2 \AA$ ) and the vinyl group (ca. $3 \AA$ ), whereas methyl 2 is pointing away from them, resulting in a higher internal rotation barrier for methyl 3 than for methyl 2. The internal rotation barrier was determined with lower accuracy in the case of methyl 3 since only a few AA and AE splittings could be resolved in our spectrum (the predicted frequency difference was less than $10 \mathrm{kHz}$ for the not resolved ones). The internal rotation barrier for methyl 2 is more dependent on the well resolved E state splittings, that is, $\mathrm{AA}, \mathrm{AE} v s . \mathrm{EA}, \mathrm{EE}^{+}$and $\mathrm{EE}^{-}$.

The internal dynamics of linalool (conformer I) is similar to the one recently described for pulegone, another terpene. ${ }^{16}$ Pulegone also has two non-equivalent methyl groups attached to the same $\mathrm{sp}^{2}$ hybridized carbon atom, whose experimental internal rotation barriers are 1.961911(46) $\mathrm{kJ} / \mathrm{mol}$ and $6.3617(12) \mathrm{kJ} / \mathrm{mol}$ for the most stable conformer (Chair 1) and similar ones for the second lowest-energy conformer (Chair 2). In this case, the multiplet structures were also composed of five components ( $\mathrm{AA}, \mathrm{AE}, \mathrm{EA}, \mathrm{EE}^{+}$and $\mathrm{EE}^{-}$), with $\mathrm{AA}$ and $\mathrm{AE}$ appearing very close and being not resolved, like in the linalool case. Interestingly, the experimental internal rotation barriers for linalool are higher than for pulegone. This might be due to an interaction with the $-\mathrm{OH}$ group in linalool, which is only $3.3 \AA$ (O-H ${ }^{\cdots} \mathrm{C}_{\text {methyl }}$ distance) away from the methyl groups (see Fig. 3 ).

\section{Conclusions}

In the present work, the abundances of the structurally related species present in two natural thyme essential oils from Spain were estimated in a semi-quantitative way, based on intensity measurements of selected rotational transitions. Structurally related species like conformers, structural isomers, and isotopologues of related terpene molecules are easily differentiated because of their different rotational spectra, making broadband rotational spectroscopy a powerful, but yet not fully explored tool to qualitatively analyze the composition of such complex molecular mixtures.

In thyme I oil, thymol is determined to be the most abundant terpene, with trans-thymol-A being the most abundant conformer, respectively, whereas for thyme II, the most abundant 
conformer is the conformer I of linalool, and the most abundant terpene is 4-carvomenthenol (occurring as three conformers). The main contribution to the error of the abundance determination arises from the uncertainty of the calculated dipole moment components. In addition, linalool has two non-equivalent methyl tops located in different chemical environments that are attached to a $\mathrm{sp}^{2}$-hybridized carbon atom, giving rise to rich internal dynamics that have been analyzed in detail in this work, extending existing work describing the internal dynamics of one methyl top. The accurate carbon backbone structures of trans-thymol-A and linalool were experimentally determined through the assignment of all their singly substituted ${ }^{13} \mathrm{C}$ species and compared to the results of quantum-chemical calculations.

In summary, broadband rotational spectroscopy can disentangle the complex composition of biologically relevant samples such as natural essential oils due to its inherent fingerprint character. For a more quantitative determination of the composition, a larger subset of rotational transitions could be considered together with a calibration of the spectrometer components using a spectrometer function to account for different excitation power and detection capabilities for the respective frequencies. With broadband rotational spectroscopy in hand, we thus do not only obtain a chemical analysis of a complex sample, but also accurate structural information for the respective species, their conformational landscape, and the internal dynamics. One further step would be to analyze the chirality of these kind of complex mixtures in the gas phase using a combination of broadband rotational spectroscopy and the chirality-sensitive microwave three-wave-mixing (M3WM) technique. ${ }^{7,18,19}$

\section{Acknowledgements}

This work is supported via the collaborative linkage grant "Extreme light for sensing and driving molecular chirality (ELCH)", SFB1319, of the Deutsche Forschungsgemeinschaft. Use of the GWDG computing cluster is acknowledged. M.M.Q.M. thanks Fundación Alfonso Martín Escudero for a postdoctoral grant. A.K. acknowledges the financial support of the IMPRS-UFAST.

\section{References}

1 C. Ballester-Costa, E. Sendra, J. Fernández-López, J. A. Pérez-Álvarez, M. Viuda-Martos, Ind. Crops Prod. 2013, 50, 304-311.

2 A. Elaissi, Z. Rouis, N. A. B. Salem, S. Mabrouk, Y. Salem, K. B. H. Salah, M. Aouni, F. Farhat, R. Chemli, F. Harzallah-Skhiri, M. L. Khouja, BMC Complement. Altern. Med. 2012, 12, 81 .

3 O. Borugă, C. Jianu, C. Mişcă, I. Goleţ, A. T. Gruia, F. G. Horhat, J. Med. Life 2014, 7, 5660 .

4 L. Stella Nerio, J. Olivero-Verbel, E. Stashenko, Bioresour. Technol. 2010, 101, 372-378.

5 D. Schmitz, V. A. Shubert, B. M. Giuliano, M. Schnell, J. Chem. Phys. 2014, 141, 034304.

6 V. A. Shubert, D. Schmitz, C. Medcraft, A. Krin, D. Patterson, J. M. Doyle, M. Schnell, J. Chem. Phys. 2015, 142, 214201.

7 V. A. Shubert, D. Schmitz, C. Pérez, C. Medcraft, A. Krin, S. R. Domingos, D. Patterson, M. Schnell, J. Phys. Chem. Lett. 2016, 7, 341-350.

$8 \quad$ P. J. Marriott, R. Shellie, C. Cornwell, J. Chromatogr. A 2001, 936, 1-22.

9 H. Schulz, R. Quilitzsch, H. Krüger, J. Mol. Struct. 2003, 661-662, 299-306.

10 H. V. L. Nguyen, H. Mouhib, S. Klahm, W. Stahl, I. Kleiner, Phys. Chem. Chem. Phys. 2013, 15, 10012-10018.

11 J. R. Avilés-Moreno, T. R. Huet, J. J. López González, Struct. Chem. 2013, 24,1163-1170.

12 V. A. Shubert, D. Schmitz, D. Patterson, J. M. Doyle, M. Schnell, Angew. Chem. Int. Ed. 2014, 53, 1152-1155.

13 D. Loru, M. A. Bermúdez, M. E. Sanz, J. Chem. Phys. 2016, 145, 074311. 
14 C. Pérez, A. Krin, A. L. Steber, J. C. López, Z. Kisiel, M. Schnell, J. Phys. Chem. Lett. 2016, 7, 154-160.

15 Z. Kisiel, O. Desyatnyk, E. Białkowska-Jaworska, L. Pszczjłkowski, Phys. Chem. Chem. Phys. 2003, 5, 820-826.

16 A. Krin, C. Pérez, P. Pinacho, M. M. Quesada-Moreno, J. J. López-González, J. R. AvilésMoreno, S. Blanco, J. Carlos López, M. Schnell, Chem. Eur. J. 2018, 24, 721-729.

17 V. A. Shubert, D. Schmitz, M. Schnell, J. Mol. Spectrosc. 2014, 300, 31-36.

18 S. R. Domingos, C. Pérez, M. Schnell, Annu. Rev. Phys. Chem. 2018, 69, 499-519.

19 B. H. Pate, L. Evangelisti, W. Caminati, Y. Xu, J. Thomas, D. Patterson, C. Pérez, M. Schnell, in Chiral Analysis: Advances in Spectroscopy, Chromatography and Emerging Methods, chapter 17 Quantitative Chiral Analysis by Molecular Rotational Spectroscopy (Ed.: P. L. Polavarapu), Elsevier, Amsterdam, 2018, pp. 679-730.

20 J. Kraitchman, Am. J. Phys. 1953, 21, 17-24.

21 S. T. Shipman, J. L. Neill, R. D. Suenram, M. T. Muckle, B. H. Pate, J. Phys. Chem. Lett. 2011, 2, 443-448.

22 C. Pérez, M. T. Muckle, D. P. Zaleski, N. A. Seifert, B. Temelso, G. C. Shields, Z. Kisiel, B. H. Pate, Science 2012, 336, 897-901.

23 N. A. Seifert, D. P. Zaleski, C. Pérez, J. L. Neill, B. H. Pate, M. Vallejo-López, A. Lesarri, E. J. Cocinero, F. Castaño, I. Kleiner, Angew. Chem. 2014, 126, 3274-3277.

24 S. Blanco, P. Pinacho, J. C. López, J. Phys. Chem. Lett. 2017, 8, 6060-6066.

25 M. Schnell, U. Erlekam, P. R. Bunker, G. von Helden, J.-U. Grabow, G. Meijer, A. van der Avoird, Angew. Chem. Int. Ed. 2013, 52, 5180-5183.

26 J. O. Richardson, C. Pérez, S. Lobsiger, A. A. Reid, B. Temelso, G. C. Shields, Z. Kisiel, D. J. Wales, B. H. Pate, S. C. Althorpe, Science 2016, 351, 1310-1313.

27 L. Evangelisti, K. Brendel, H. Mäder, W. Caminati, S. Melandri, Angew. Chem. 2017, 129, 13887-13891.

28 A. Jabri, V. Van, H. V. L. Nguyen, H. Mouhib, F. Kwabia Tchana, L. Manceron, W. Stahl, I. Kleiner, Astron. Astrophys. 2016, A127, 1-13.

29 L. Ferres, J. Cheung, W. Stahl, H. V. L. Nguyen, J. Phys. Chem. A 2019, 123, 3497-3503.

30 K. P. R. Naira, S. Herbers, A. Lesarri, J.-U. Grabow, J. Mol. Spectrosc. 2019, 361, 1-7.

31 https://www.florihana.com/en/90-essential-oils

32 D. Schmitz, V. A. Shubert, T. Betz, M. Schnell, J. Mol. Spectrosc. 2012, 280, 77-84.

33 C. Pérez, S. Lobsiger, N. A. Seifert, D. P. Zaleski, B. Temelso, G. C. Shields, Z. Kisiel, B. H. Pate, Chem. Phys. Lett. 2013, 571, 1-15.

34 D. Plusquellic, JB95, available at http://www.nist.gov/pml/electromagnetics/grp05/jb95.cfm

35 C. M. Western, J. Quant. Spectrosc. Radiat. Transfer 2017, 186, 221-242.

36 H. M. Pickett, J. Mol. Spectrosc. 1991, 148, 371-377.

37 H. Hartwig, H. Dreizler, Z. Naturforsch. A 1996, 51, 923-932.

38 R. C. Woods, J. Mol. Spectrosc. 1966, 21, 4-24.

39 R. C. Woods, J. Mol. Spectrosc. 1967, 22, 49-59.

40 M. Clark, R. D. Cramer III, N. V. Opdensch, J. Comput. Chem. 1989, 10, 982-1012.

41 Spartan'16 for Linux. Wavefunction.

42 Gaussian 09 (Revision E.01), M. J. Frisch, G. W. Trucks, H. B. Schlegel, G. E. Scuseria, M. A. Robb, J. R. Cheeseman, G. Scalmani, V. Barone, B. Mennucci, G. A. Petersson, H. Nakatsuji, M. Caricato, X. Li, H. P. Hratchian, A. F. Izmaylov, J. Bloino, G. Zheng, J. L. Sonnenberg, M. Hada, M. Ehara, K. Toyota, R. Fukuda, J. Hasegawa, M. Ishida, T. Nakajima, Y. Honda, O. Kitao, H. Nakai, T. Vreven, J. A. Montgomery, J r., J. E. Peralta, F. Ogliaro, M. Bearpark, J. J. Heyd, E. Brothers, K. N. Kudin, V. N. Staroverov, R. Kobayashi, J. Normand, K. Raghavachari, A. Rendell, J. C. Burant, S. S. Iyengar, J. Tomasi,M.Cossi, N. Rega, J. M. Millam, M. Klene, J. E. Knox, J. B. Cross, V. Bakken, C. Adamo, J. Jaramillo, R. 
Gomperts, R. E. Stratmann, O. Yazyev, A. J. Austin, R. Cammi, C. Pomelli, J. W. Ochterski, R. L. Martin, K. Morokuma, V. G. Zakrzewski, G. A. Voth, P. Salvador, J. J. Dannenberg, S. Dapprich, A. D. Daniels, O. Farkas, J. B. Foresman, J. V. Ortiz, J. Cioslowski, D. J. Fox, Gaussian Inc. Wallingford CT 2009.

43 J. C. McGurk, T. G. Schmalz, W. H. Flygare, J. Chem. Phys. 1974, 60, 4181-4188.

44 S. T. Shipman, B. H. Pate in Handbook of High-resolution Spectroscopy, chapter New techniques in microwave spectroscopy (Ed.: M. Quack, F. Merkt), John Wiley \& Sons, 2011, pp. 801-827.

45 A. L. Hickey, C. N. Rowley, J. Phys. Chem. A 2014, 118, 3678-3687.

46 E. Kraka, J. Gauss, D. Cremer, J. Mol. Struct.-Theochem 1991, 234, 95-126.

47 V. I. Minkin, O. A. Osipov, Y. A. Zhdanov in Dipole Moments in Organic Chemistry, series Physical Methods in Organic Chemistry, Springer, New York, 2012, pp. 69-71.

48 Z. Kisiel, PROSPE Programs for ROtational SPEctroscopy, available at http://www.ifpan.edu.pl/ kisiel/prospe.htm

49 C. C. Costain, Trans. Am. Crystallogr. Assoc. 1966, 2, 157-164.

50 S. R. Domingos, C. Pérez, C. Medcraft, P. Pinacho, M. Schnell, Phys. Chem. Chem. Phys., 2016, 18, 16682-16689.

51 H. Dreizler, Z. Naturforsch. 1961, 16a, 1354-1367.

52 J. Meier, A. Bauder, Hs. H. Günthard, J. Chem. Phys. 1972, 57, 1219-1236. 